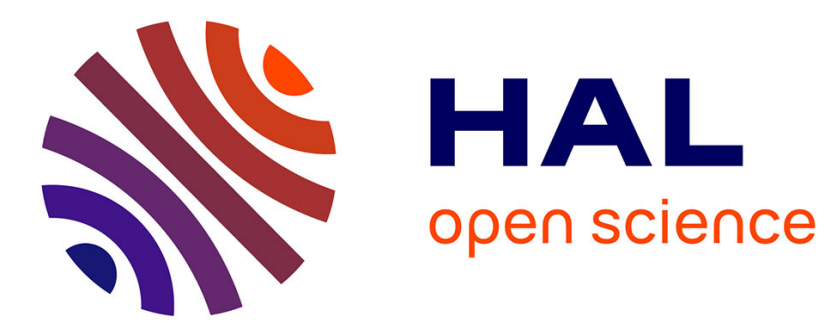

\title{
On (Weighted) k-Order Fuzzy Connectives
}

Hoel Le Capitaine, Carl Frélicot

\section{To cite this version:}

Hoel Le Capitaine, Carl Frélicot. On (Weighted) k-Order Fuzzy Connectives. IEEE Int. Conf. on Fuzzy Systems, 2010, Spain. pp.1-8. hal-00745292

\section{HAL Id: hal-00745292 \\ https://hal.science/hal-00745292}

Submitted on 25 Oct 2012

HAL is a multi-disciplinary open access archive for the deposit and dissemination of scientific research documents, whether they are published or not. The documents may come from teaching and research institutions in France or abroad, or from public or private research centers.
L'archive ouverte pluridisciplinaire HAL, est destinée au dépôt et à la diffusion de documents scientifiques de niveau recherche, publiés ou non, émanant des établissements d'enseignement et de recherche français ou étrangers, des laboratoires publics ou privés. 


\title{
On (Weighted) $k$-Order Fuzzy Connectives
}

\author{
Hoel Le Capitaine and Carl Frélicot \\ Mathematics, Image and Applications (MIA) \\ Université de La Rochelle Avenue M. Crépeau, \\ 17042 La Rochelle Cedex 1, FRANCE, \\ email:\{firstname.lastname\}@univ-lr.fr
}

\begin{abstract}
In this paper, we present new fuzzy connectives that allow to specify an order to the considered operation. These operators are generalization of usual fuzzy connectives, i.e. triangular norms and triangular conorms. A potential use of the proposed operators consists in assessing to what extent several values are high or low in unconstrained fuzzy sets is given. We also present weighted $k$-order fuzzy connectives, where weights are associated to different subsets of criteria. Finally, we show that these fuzzy connectives can be used from a set-theoretic point of view, enabling to define new kinds of fuzzy intersection and union.
\end{abstract}

\section{INTRODUCTION}

The aggregation of information is an important issue of intelligent decision systems design. In such systems, the data to be analyzed often presents some kind of imprecision, so that tools taking into account of this lack of knowledge are needed. To this aim, numerous aggregation operators were introduced in the last decades. Beyond them, one can find the mean and its derivatives (e.g. quasi-arithmetic means, OWA operators), fuzzy integrals, or the triangular norms, see [1] for a large survey. Here, we consider the problem of taking a decision while having some uncertainty regarding the information at hand. In particular, we are interested in defining measures which evaluate the degree to which statements are satisfied, and how many. Most of works in this area are based on quantifiers the user must specify [2], [3], [4]. Our proposition is to allow the user to only specify the number of required satisfactions or dissatisfactions. A natural way to do this is to use a convenient combination of conjunctive and disjunctive operators such as triangular norms.

The paper is organized as follows. In Section II, we recall some fundamentals of triangular norms and the disjunctive $k$-order operator, namely the $k$-order fuzzy $O R$. We then propose its dual conjunctive operator, called the $k$-order fuzzy $A N D$, and present how it can be used to seek for high or low values in a set of values representing satisfaction degrees in Section III. The derived weighted $k$-order connectives are proposed in Section IV. Operations on fuzzy sets based on the new operators are presented in Section V. Concluding remarks and perspectives are given in Section VI.

\section{Preliminaries}

\section{A. Triangular Norms}

The family of triangular norms, first introduced by Menger [5] to generalize the triangle inequality of ordinary metric spaces, has been used to characterize the generalized multivalued logic $A N D$ and $O R$ operations in lattices, the conjunction and disjunction in fuzzy logic. We briefly introduce these operators in the sequel. First, we shall split this family into two members: the triangular norms ( $t$-norms for short) and triangular conorms ( $t$-conorms for short), corresponding to intersection/conjunction, and union/disjunction operations, respectively. A $t$-norm is a function $T:[0,1]^{2} \rightarrow[0,1]$ satisfying: for any $x, y$ and $z$ in $[0,1]$

(T1) commutativity: $\mathrm{\top}(x, y)=\top(y, x)$,

(T2) monotonicity: $\top(x, y) \geq \top(x, z)$ if $y \geq z$,

(T3) associativity: $\top(x, \top(y, z))=\top(\top(x, y), z)$,

(T4) 1 as neutral element: $\top(x, 1)=x$.

The associativity (T3) allows to extend a $t$-norm $T$ to a $n$-ary operator. Another representation theorem allows to compute $n$-ary $t$-norms in an easy and efficient way thanks to additive or multiplicative generators [6]. Given the strictly decreasing additive generator $f:[0,1] \rightarrow[0, \infty]$, such as $f(1)=0$, then the $t$-norm $\top$ is defined by

$$
\top\left(x_{1}, \cdots, x_{n}\right)=f^{(-1)}\left(\sum_{i=1}^{n} f\left(x_{i}\right)\right) .
$$

where $f^{(-1)}$ is the pseudo-inverse of $f$. In the sequel, we will denote the $n$-tuples $\left\{x_{1}, \ldots, x_{n}\right\}$ and $\left\{1-x_{1}, \ldots, 1-x_{n}\right\}$ as $\mathbf{x}$ and $\mathbf{1}-\mathbf{x}$ respectively. The dual operation of a $t$-norm is a $t$-conorm $\perp:[0,1]^{2} \rightarrow[0,1]$ satisfying (T1), (T2) and (T3), and (T4') instead of (T4):

(T4') 0 as neutral element: $\perp(x, 0)=x$.

The four basic norm couples $(\top, \perp)$ are given in Table I.

\section{B. The k-Order Fuzzy OR}

In many decision making problems, one may want to be able to determine whether all, a part or none of individual scores are satisfied, from subsets representing satisfactions to criteria (say $\mathbf{x}$ ). The easiest way to determine if none of the scores are satisfied is to compute the $t$-conorm $\perp(\mathbf{x})$. If 
TABLE I

FOUR PROTOTYPICAL EXAMPLES OF TRIANGULAR NORMS.

\begin{tabular}{|c|c|c|c|}
\hline \multirow{2}{*}{ Standard } & \multicolumn{3}{|c|}{$\top_{M}(x, y)=\min (x, y)$} \\
\hline & \multicolumn{3}{|c|}{$\perp_{M}(x, y)=\max (x, y)$} \\
\hline \multirow{2}{*}{ Product } & \multicolumn{3}{|c|}{$\top_{P}(x, y)=x y$} \\
\hline & \multicolumn{3}{|c|}{$\perp_{P}(x, y)=x+y-x y$} \\
\hline \multirow{2}{*}{ Łukasiewicz } & \multicolumn{3}{|c|}{$\top_{L}(x, y)=\max (x+y-1,0)$} \\
\hline & \multicolumn{3}{|c|}{$\perp_{L}(x, y)=\min (x+y, 1)$} \\
\hline \multirow[t]{2}{*}{ Drastic } & $\top_{D}(x, y)=\{$ & $\begin{array}{l}0 \\
\min (x, y)\end{array}$ & $\begin{array}{l}\text { if }(x, y) \in\left[0,1\left[^{2}\right.\right. \\
\text { otherwise }\end{array}$ \\
\hline & $\perp_{D}(x, y)=\{$ & $\begin{array}{l}1 \\
\max (x, y)\end{array}$ & $\begin{array}{l}\text { if }(x, y) \in] 0,1]^{2} \\
\text { otherwise }\end{array}$ \\
\hline
\end{tabular}

it is high ${ }^{1}$, then at least one of them is high. Inversely, if one wants to determine if all the scores are high, the use of a $t$-norm is preferable. The value of $T(\mathbf{x})$ will be high if all of the individual scores are high. By duality of $T$ and $\perp$, the user can easily evaluate if at least one value is low or if all the scores are low by respectively computing $\perp(1-\mathbf{x})$ and $T(1-\mathbf{x})$.

In [7], the authors introduce the $k$-order fuzzy $O R(f O R-k)$, which is a conjunctive combination of the disjunctive combinations applied to specific subsets of $\mathbf{x}$. Let $\mathcal{P}$ be the power set of $N=\{1,2, \ldots, n\}$ and $\mathcal{P}_{k}=\{A \in \mathcal{P}: \operatorname{card}(A)=k\}^{2}$, it is defined by

$$
\stackrel{k}{\perp}(\mathbf{x})=\stackrel{k}{\perp}_{i=1, n}^{k} x_{i}=\prod_{A \in \mathcal{P}_{k-1}}\left(\underset{j \in N \backslash A}{\perp} x_{j}\right) .
$$

This operator shows nice properties such as symmetry, boundary conditions, monotonicity, allowing us to qualify $k$

$\perp(\mathbf{x})$ as an aggregation operator. Additional properties are of particular interest, see [7] for proofs:

- $\stackrel{1}{\perp}(\mathbf{x})=\perp(\mathbf{x})$ and $\stackrel{n}{\perp}(\mathbf{x})=\top(\mathbf{x})$,

- $\perp$ ( $\mathbf{x})$ is exactly the $k^{\text {th }}$ highest element of $\mathbf{x}$ if the standard norms ( $\top_{M}=\min , \perp_{M}=\max$ ) are taken.

This operator aims at evaluating if $k$ values of $\mathbf{x}$ are high, and has been used in pattern recognition problems, e.g. reject options in pattern classification [7] and cluster validity [8].

\section{THE $k$-ORDER FUZZY AND}

\section{A. Motivation}

In this paper, our goal is to determine how many values are high or low? For the special case of exactly one high value, some propositions have been made, let us cite the fuzzy exclusive OR operator [9], or specificity measures [4].

\footnotetext{
${ }^{1}$ we assume that $a \in[0,1]$ is high if $a \geq N e g(a)$, i.e. $a$ is more satisfied than not $a$, and that $a$ is low if it is not high

${ }^{2} \operatorname{card}(A)$ is the usual cardinality of the crisp set $A$
}

Another attempt can be found in [3], which consists, for reinforcement purpose, in specifying fuzzy quantifiers to obtain a lower bound on the high scores. Finally, in [2], still with fuzzy quantifiers, the authors propose a measure which enable to determine if a fuzzy set contains $k$ elements, but without specifying if they are high or low (implicitly, they consider the high case).

\section{B. Definition and Properties}

We introduce the dual operator of the $k$-order fuzzy $O R$, allowing us to evaluate if $k$ values of $\mathbf{x}$ are low.

Definition 1. Let $\stackrel{k}{\perp}(\mathbf{x})$ be the $k$-order fuzzy $O R$, then its dual operator, called the $k$-order fuzzy $A N D$ is given by

$$
\stackrel{k}{\top}(\mathbf{x})=1-\stackrel{k}{\perp}(1-\mathbf{x}) .
$$

Proposition 1. By duality to (2), $\stackrel{k}{\top}(\mathbf{x})$ is

$$
\prod_{i=1, n}^{k} x_{i}=\underset{A \in \mathcal{P}_{k-1}}{\perp}\left(\prod_{j \in N \backslash A} x_{j}\right)
$$

Proof:

$$
\begin{aligned}
T^{k}(\mathbf{x}) & =1-\perp^{k}(1-\mathbf{x}) \\
& =1-\prod_{A \in \mathcal{P}_{k-1}}\left(\underset{j \in N \backslash A}{\perp}\left(1-x_{j}\right)\right) .
\end{aligned}
$$

Since $\perp(x, y)=1-\top(1-x, 1-y)$, we obtain

$$
\begin{aligned}
T^{k}(\mathbf{x}) & =1-\prod_{A \in \mathcal{P}_{k-1}}\left(1-\prod_{j \in N \backslash A} x_{j}\right) \\
& =1-\left(1-\underset{A \in \mathcal{P}_{k-1}}{\perp}\left(\prod_{j \in N \backslash A} x_{j}\right)\right) \\
& =\underset{A \in \mathcal{P}_{k-1}}{ }\left(\prod_{j \in N \backslash A} x_{j}\right) .
\end{aligned}
$$

In the sequel, we will use ${ }^{k}(\mathbf{x})$ as defined by (4). Let us present the properties the $k$-order fuzzy AND (fAND- $k$ for short) holds.

Theorem 1. The $f A N D-k$ operator (4) is an aggregation operation in the sense that it satisfies the following properties:

(P1) boundary condition: ${ }^{k}(\mathbf{0})=0$ and ${ }^{k}(\mathbf{1})=1$,
(P2) monotony: if $\mathbf{x} \leq \mathbf{y}$, then $T(\mathbf{x}) \leq T(\mathbf{y}) .^{3}$

for all norm couple $(\top, \perp)$.

${ }^{3} \mathbf{x} \leq \mathbf{y}$ if $x_{i} \leq y_{i}, \forall i \in[1, n]$ 
Theorem 2. The $f A N D-k$ aggregation operator (4) is symmetric:

$$
\prod_{i=1, n}^{k} x_{i}=\prod_{i=1, n}^{k} x_{\sigma(i)}
$$

for any permutation $\sigma(\cdot)$.

Proofs of theorems 1 and 2 are straightforward and are left for a forthcoming journal paper. From an application point of view, symmetry is often required because it means that the values to be aggregated are "anonymous" with respect to the operator.

The following property addresses the use of standard norms $\left(\top_{M}=\min , \perp_{M}=\max \right)$. In this case, fAND- $k$ reduces to the $k^{\text {th }}$-order statistic, which is widely used in non-parametric statistics and inference.

Theorem 3. When using $T=\min$ and $\perp=\max$, the $k$-order fuzzy $A N D$ is exactly the $k^{\text {th }}$-order statistic, i.e. $\stackrel{k}{\top}(\mathbf{x})=x_{(n-k+1)}$, where $(\cdot)$ is a permutation on $\mathbf{x}$ such that $x_{(1)} \geq x_{(2)} \geq \cdots \geq x_{(n)}$.

\section{Proof:}

Let us decompose the $f A N D-k$ operator (4) as

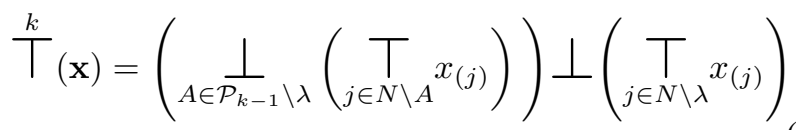

where $\lambda=\{n-k+2, \cdots, n\}$.

In the general case, we have $T_{j \in N \backslash \lambda} x_{(j)} \leq x_{(n-k+1)}$, which reduces to $\rceil_{j \in N \backslash \lambda} x_{(j)}=x_{(n-k+1)}$ when using min for $\top$. Consequently, the only necessary condition to complete the proof is to show that the first term of (5) is lower or equal than $x_{(n-k+1)}$ as follows.

If $A \in \mathcal{P}_{k-1} \backslash \lambda$, then the subset $\lambda \cap(N \backslash A)$ is not empty. Let us denote $\ell$ the lowest index of this subset. Then we can write, for all $A \in \mathcal{P}_{k-1} \backslash \lambda$ :

$$
\rceil_{j \in N \backslash A} x_{(j)} \leq x_{(\ell)} \leq x_{(n-k+1)} .
$$

It follows that, when using max for $\perp$ :

$$
\left.\left(\underset{A \in \mathcal{P}_{k-1} \backslash \lambda}{\perp}(\rceil_{j \in N \backslash A} x_{(j)}\right)\right) \leq x_{(n-k+1)},
$$

which concludes the proof.

Theorem 4. Whatever the norm couple $(\top, \perp)$, the $k$-order fuzzy $A N D$ is bounded from above and below by $\top$ and $\perp$ respectively: $\forall k \in N=\{1, \ldots, n\}$,

$$
\top(\mathbf{x}) \leq \stackrel{k}{\top}(\mathbf{x}) \leq \perp(\mathbf{x})
$$

Moreover: $\stackrel{1}{\top}(\mathbf{x})=\top(\mathbf{x})$ and $\stackrel{n}{\top}(\mathbf{x})=\perp(\mathbf{x})$.

Proof:

Let us first consider the case $1<k<n$, and prove the left-hand side inequality. Taking the decomposition (5) of fAND- $k$ with $\lambda=\{1, \cdots, k-1\}$, we get:

$$
\begin{aligned}
T^{k}(\mathbf{x}) & =\left(\underset{A \in \mathcal{P}_{k-1} \backslash \lambda}{\perp}\left(\prod_{j \in N \backslash A} x_{(j)}\right)\right) \perp\left(\prod_{j \in\{k, \cdots, n\}} x_{(j)}\right) \\
& \geq\rceil_{j \in\{k, \cdots, n\}} x_{(j)} \\
& \geq \underset{j \in\{1, \cdots, n\}}{T_{(j)}} x_{(j,}=\top(\mathbf{x})
\end{aligned}
$$

by monotony, associativity and commutativity of $t$-norms. The proof of the right-hand side inequality is slightly different. We have, for all $i \in N$ :

$$
\left.\left.x_{(i)} \geq x_{(i)} \top(\rceil_{j \in N \backslash A, j \neq i} x_{(j)}\right)=\right\rceil_{j \in N \backslash A} x_{(j)} .
$$

By monotony, associativity and commutativity of $t$-conorms, we have:

$$
\begin{gathered}
\underset{i \in \mathcal{P}_{k-1}}{\perp} x_{(i)} \geq \underset{A \in \mathcal{P}_{k-1}}{\perp}\left(\prod_{j \in N \backslash A} x_{j}\right) \\
\text { and } \quad \frac{1}{i \in N} x_{(i)} \geq \underset{i \in \frac{1}{\mathcal{P}_{k-1}}}{ } x_{(i)},
\end{gathered}
$$

which leads to $\perp(\mathbf{x}) \geq T^{k}(\mathbf{x})$ and thus concluding the first part of the proof.

If $k=1$, then ${ }^{\top}(\mathbf{x})$ can be rewritten as

$$
\frac{\perp}{A \in \mathcal{P}_{0}}\left(\prod_{j \in N \backslash A} x_{j}\right) \text {. }
$$

Since $\mathcal{P}_{0}$ is the empty set $\emptyset$, it follows that ${ }^{\top}(\mathbf{x})=\prod_{j \in N} x_{j}$, which is $T(\mathbf{x})$.

If $k=n$, then we have:

$$
\begin{aligned}
T^{n}(\mathbf{x}) & =\underset{A \in \mathcal{P}_{n-1}}{\perp}\left(\prod_{j \in N \backslash A} x_{j}\right) \\
& =\underset{A \in \mathcal{P}_{n-1}}{\perp}\left(\prod_{j \in \mathcal{P}_{1}} x_{j}\right)
\end{aligned}
$$

which is equal to $\frac{\perp}{j \in N} x_{j}$ because $\operatorname{card}\left(\mathcal{P}_{n-1}\right)=n$, therefore to $\perp(\mathbf{x})$. 
Given $\mathbf{x}, \stackrel{k}{\top}(\mathbf{x})$ is low if $k$ values in $\mathbf{x}$ are low. If one wants to get the truth value of the statement $k$ values in $\mathbf{x}$ are low, the strict negation $\left(1-\frac{k}{T}(\mathbf{x})\right)$ must be taken.

\section{Examples}

We first exemplify the $k$-order fuzzy connectives computation using the standard norm couple $(\top, \perp)_{M}$. Let us take $n=4$ so that $\mathbf{x}=\left(x_{1}, x_{2}, x_{3}, x_{4}\right)$ and $N=\{1,2,3,4\}$, and let us set $k=3$ so that $\mathcal{P}_{2}=\{\{1,2\},\{1,3\},\{1,4\},\{2,3\},\{2,4\},\{3,4\}\}$.

Without loss of generality, let us assume that $\mathrm{x}$ is sorted such as $x_{(1)} \geq x_{(2)} \geq x_{(3)} \geq x_{(4)}$. Selecting subsets which are not in $\mathcal{P}_{2}$ gives:

$$
\begin{aligned}
& \stackrel{3}{\perp}(\mathbf{x})= \min \left(\max \left(x_{(3)}, x_{(4)}\right), \max \left(x_{(2)}, x_{(4)}\right), \max \left(x_{(2)}, x_{(3)}\right),\right. \\
&\left.\max \left(x_{(1)}, x_{(4)}\right), \max \left(x_{(1)}, x_{(3)}\right), \max \left(x_{(1)}, x_{(2)}\right)\right) \\
&= \min \left(x_{(3)}, x_{(2)}, x_{(2)}, x_{(1)}, x_{(1)}, x_{(1)}\right) \\
&= x_{(3)}=x_{(k)} \text { and } \\
& \stackrel{3}{\top}(\mathbf{x})=\max \left(\min \left(x_{(3)}, x_{(4)}\right), \min \left(x_{(2)}, x_{(4)}\right), \min \left(x_{(2)}, x_{(3)}\right),\right. \\
&\left.\quad \min \left(x_{(1)}, x_{(4)}\right), \min \left(x_{(1)}, x_{(3)}\right), \min \left(x_{(1)}, x_{(2)}\right)\right) \\
&=\max \left(x_{(4)}, x_{(4)}, x_{(3)}, x_{(4)}, x_{(3)}, x_{(2)}\right) \\
&=x_{(2)}=x_{(n-k+1) .} .
\end{aligned}
$$

Next, we show how the $k$-order fuzzy connectives behave with respect to two different norm couples of Table I for the following examples of sets of satisfaction degrees, assumed to be given by $n=5$ experts:

- $\mathbf{x}=\{0.9,0.8,0.3,0.2,0.1\}$ composed of two high and three low values, meaning that the experts clearly do not agree with each other,

- $\mathbf{y}=\{0.97,0.81,0.83,0.87,0.91\}$ representing a fully agreement on acceptation, and

- $\mathbf{z}=\{0.15,0.20,0.09,0.05,0.09\}$ representing a fully agreement on rejection.

The values of the two fuzzy connectives of interest, namely $\stackrel{k}{\perp}(\mathbf{x})$ and $\left(1-{ }^{k}(\mathbf{x})\right)$, are given in Table II, where high values (say $>0.5$ ) are in bold. From this table, one can see that using both $(\top, \perp)_{M}$ and $(\top, \perp)_{P}$ give the expected results:

\begin{tabular}{|c|c|c|c|c|c|c|}
\hline \multirow[t]{2}{*}{$\mathbf{x}$} & & \multicolumn{5}{|c|}{$k$} \\
\hline & connectives & 1 & 2 & 3 & 4 & 5 \\
\hline \multirow{2}{*}{$(\top, \perp)_{M}$} & $1-\frac{k}{\top}(\mathbf{x})$ & 0.900 & 0.800 & 0.700 & 0.200 & 0.100 \\
\hline & $\begin{array}{l}k \\
\perp\end{array}(\mathbf{x})$ & 0.900 & 0.800 & 0.300 & 0.200 & 0.100 \\
\hline \multirow{2}{*}{$(\top, \perp)_{P}$} & $1-\frac{k}{\top}(\mathbf{x})$ & 0.995 & 0.913 & 0.511 & 0.080 & 0.010 \\
\hline & $\begin{array}{l}k \\
\perp \\
(\mathbf{x})\end{array}$ & 0.989 & 0.821 & 0.257 & 0.020 & 0.004 \\
\hline
\end{tabular}

- $\stackrel{k}{\perp}(\mathbf{x})$ are high up to $k=2(0.800$ and 0.821$)$ and

\begin{tabular}{|c|c|c|c|c|c|c|}
\hline $\mathbf{y}$ & & & & $k$ & & \\
\hline & connectives & 1 & 2 & 3 & 4 & 5 \\
\hline \multirow{2}{*}{$(\top, \perp)_{M}$} & $1-\frac{k}{\top}(\mathbf{y})$ & 0.190 & 0.170 & 0.130 & 0.090 & 0.030 \\
\hline & $\perp(\mathbf{y})$ & 0.970 & 0.910 & 0.870 & 0.830 & 0.810 \\
\hline \multirow{2}{*}{$(\top, \perp)_{P}$} & $1-\frac{k}{\top}(\mathbf{y})$ & 0.483 & 0.011 & 0.002 & 0.000 & 0.000 \\
\hline & $\perp(\mathbf{y})$ & 1.000 & 0.999 & 0.985 & 0.867 & 0.516 \\
\hline
\end{tabular}
$\left(1-\frac{k}{\top}(\mathbf{x})\right)$ are high up to $k=3(0.700$ and 0.511$)$, $\stackrel{k}{\perp}(\mathbf{y})$ are high, i.e. up to $k=5$ (0.810 and 0.516) and all $\left(1-\frac{k}{\top}(\mathbf{y})\right)$ are low,
TABLE II

EXAMPLES OF $k$-ORDER FUZZY CONNECTIVES VALUES (HIGH VALUES ARE IN BOLD).

\begin{tabular}{|c|c|ccccc|}
\hline $\mathbf{z}$ & & \multicolumn{5}{|c|}{$k$} \\
\hline & connectives & 1 & 2 & 3 & 4 & 5 \\
\hline \multirow{2}{*}{$(, \perp)_{M}$} & $\begin{array}{c}1-\frac{k}{\top}(\mathbf{z}) \\
\stackrel{\mathbf{0 . 9 5 0}}{(\mathbf{z})}\end{array}$ & 0.200 & 0.150 & 0.090 & 0.090 & 0.050 \\
\hline \multirow{2}{*}{$(\top, \perp)_{P}$} & $\begin{array}{c}\left.1-\frac{\mathbf{0 . 9 1 0}}{}+\mathbf{z}\right) \\
\perp(\mathbf{z})\end{array}$ & $\mathbf{1 . 0 0 0}$ & $\mathbf{0 . 9 9 9}$ & $\mathbf{0 . 9 8 6}$ & $\mathbf{0 . 8 7 9}$ & $\mathbf{0 . 5 3 5}$ \\
& 0.465 & 0.009 & 0.000 & 0.000 & 0.000 \\
\hline
\end{tabular}

- $(1-\underset{k}{k}(\mathbf{z}))$ are high, i.e. up to $k=5$ (0.800 and 0.535), and all $\perp(\mathbf{z})$ are low. Note that even if two values of $\mathbf{z}$ are identical, they are well handled by the proposed operators.

Note however that for the unanimous cases $\mathbf{y}$ and $\mathbf{z}$, the operators based on $(T, \perp)_{P}$ do not give as high (or low) values for the extreme values of $k$ under interest as one could expect. This is mainly due to the reinforcement (respectively downward and upward) of such triangular norm operators, which are retrieved for extreme values of $k$, here 1 and 5 .

Another reason is that $t$-norms, respectively $t$-conorms reinforce each other when they are combined to define the $k$ order fuzzy operators. Both reasons are accentuated for couples that are known to present a more severe reinforcement property and a warned user should revise down (respectively up) its perception of a high (respectively low) value, for instance $>0.7$ (respectively $<0.3$ ). A solution to latter problem for $t$-conorms, called noble reinforcement, has been proposed in [3]. The objective of noble reinforcement is to avoid that a collection of low values be reinforced when using $t$-conorms. The author proposes to use parametric $t$-conorms to solve the problem. Such a proposition, or the more recent one in [10] could be adopted here, but it is quite out of the scope of this paper, so it is left to be the topic of another forthcoming paper.

For illustration purpose, we give in Fig. 1 the isolevels of the proposed $k=2$-order fuzzy connectives for all 

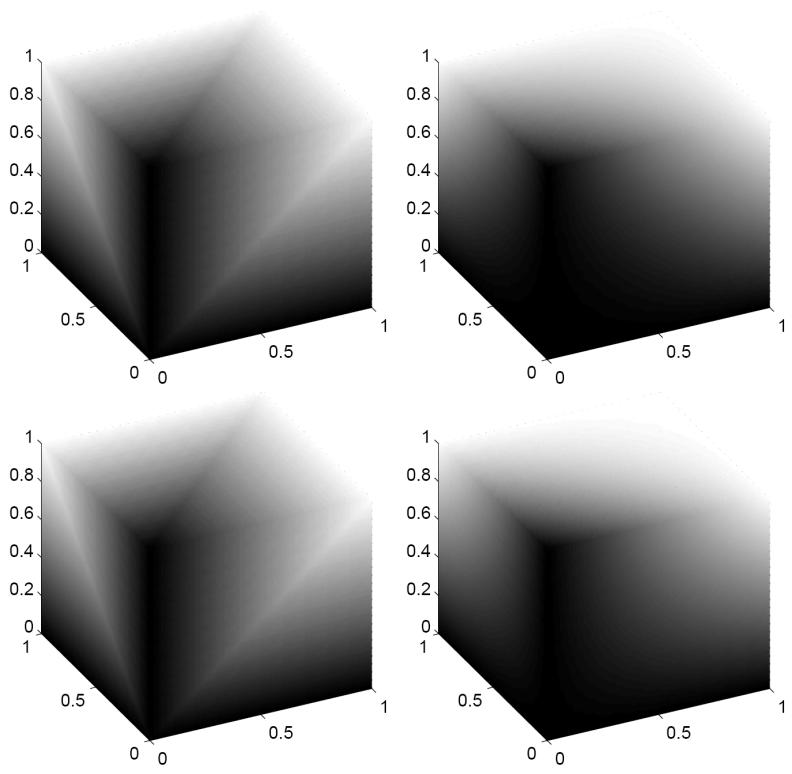

Fig. 1. fOR-2 (top) and $f A N D-2$ (bottom) operators based on standard (left) and product (right) norm couples.

$\mathbf{x} \in[0,1]^{3}$, where black (respectively white) areas indicate low (respectively high) values.

\section{WeIGHTEd $k$-Order FuZZY CONNECTIVES}

\section{A. Definitions}

It is well known that parametrization of operators can improve their performances because parameters often allows to better represent the data to be analyzed. Moreover, in many decision making systems, some criteria may not be independent, and it is useful to modelize this dependence by giving weights to subsets of criteria. To this end, we propose to extend the definition of the $k$-order fuzzy connectives. Several weighted triangular norms have been proposed so far, e.g. in [11], [12].

Let $\mathbf{w}=\left\{w_{1}, \ldots, w_{n}\right\}$ be the $n$-tuple of weights associated to the scores $\mathbf{x}$. In [12], the authors define the weighted $t$ conorm as $\perp_{\mathbf{w}}:[0,1]^{n} \rightarrow[0,1]$,

$$
\perp_{\mathbf{w}}(\mathbf{x})=\perp(\mathbf{w} \odot \mathbf{x})
$$

where $\odot$ is the binary operation $\odot:[0,+\infty[\times[0,1] \rightarrow[0,1]$, $w \odot x=\sup (y \in[0,1]: \exists i, j \in \mathbb{N}, i / j<w$ and $u \in[0,1]$

$$
\text { such that } \perp(\underbrace{u, \cdots, u}_{j \text { times }})<x \text { and } y=\perp(\underbrace{u, \cdots, u}_{i \text { times }})) \text {. }
$$

By duality, the weighted $t$-norm can be defined by

$$
\top_{\mathbf{w}}(\mathbf{x})=1-\perp_{\mathbf{w}}(1-\mathbf{x}) .
$$

For continuous Archimedean $t$-conorms, the weighted $t$ conorm can be obtained with an additive generator $g$ as follows, provided that $\mathbf{w}$ is a normal set of weights ${ }^{4}$ :

$$
\perp_{\mathbf{w}}(\mathbf{x})=g^{(-1)}\left(\sum_{i=1}^{n} w_{i} g\left(x_{i}\right)\right)
$$

where $g:[0,1] \rightarrow[0, \infty]$ is a strictly increasing function with $g(0)=0$.

If $f$ and $g$ are respectively the additive generators of $\top$ and $\perp$, then the $f O R-k$ operator 2 can be defined by:

$$
\stackrel{k}{\perp}(\mathbf{x})=f^{(-1)}\left(\sum_{A \in \mathcal{P}_{k-1}} f\left(g^{(-1)}\left(\sum_{j \in N \backslash A} g\left(x_{j}\right)\right)\right)\right)
$$

In order derive the weighted $f O R-k$, we need to introduce two weightings instead of the one $(w)$ required to define the weighted $t$-conorm (6):

$$
\begin{aligned}
& \text { - } \mathbf{v}=\left\{v_{A}: A \in \mathcal{P}_{k-1}\right\} \text { of size } \operatorname{card}\left(\mathcal{P}_{k-1}\right)=\left(\begin{array}{c}
n \\
k-1
\end{array}\right), \\
& \text { - } \mathbf{W}=\left\{w_{A, j}: A \in \mathcal{P}_{k-1}, j \in N \backslash A\right\} \text { of size } \\
& \left(\begin{array}{c}
n \\
n-k+1
\end{array}\right) \times(n-k+1) .
\end{aligned}
$$

Note that each row $\mathbf{w}_{A}$ of the array $\mathbf{W}$ corresponds to a subset $A$ of $N$, so the number or rows is exactly ${ }^{5}$ the size of $\mathbf{v}$, i.e. the number of all possible subsets of $N$ of size $k-1$, although they do not concern the same subsets. Furthermore, each $\mathbf{w}_{\mathbf{A}}$ must be normal, i.e. $\sum_{j \in N \backslash A} w_{A, j}=1$.

Definition 2. Given $f$ and $g$ two additive generators of a $t$-norm and its dual $t$-conorm, respectively, the weighted $k$ order fuzzy $O R$ is defined by:

$$
\frac{1}{\mathbf{W}, \mathbf{v}}(\mathbf{x})=f^{(-1)}\left(\sum_{A \in \mathcal{P}_{k-1}} v_{A} f\left(g^{(-1)}\left(\sum_{j \in N \backslash A} w_{A, j} g\left(x_{j}\right)\right)\right)\right)
$$

where $\mathbf{W}$ and $\mathbf{v}$ are user-defined sets of weights.

The weights in $\mathbf{W}$ and $\mathbf{w}$ can be related with capacities, in particular to $k$-order additive fuzzy measures, see [13]. Whereas the specification of a $k$-order additive fuzzy measure requires $\sum_{j=1}^{k}\left(\begin{array}{l}n \\ j\end{array}\right)$ coefficients, we only have to specify the weights (or fuzzy measures) of subsets of $N$ whose cardinality is respectively $k-1$ for $\mathbf{v}$ and $n-k+1$ for $\mathbf{W}$. Since $\mathbf{W}$ focuses on subsets which are not the ones weighted by $\mathbf{v}$, the importance given to each subset through $\mathbf{W}$ does not interfere with those given by $\mathbf{v}$, and $\mathbf{v}$ and $\mathbf{W}$ are totally different. It is only a matter of giving to all subsets of cardinality $k-1$ and $n-k+1$ a corresponding weight satisfying the normal constraints. The dual operator

$$
\begin{aligned}
& { }^{4} \mathbf{w} \text { is said to be normal if } \sum_{i=1}^{n} w_{i}=1 \\
& { }^{5} \text { indeed, }\left(\begin{array}{c}
n \\
k-1
\end{array}\right)=\left(\begin{array}{c}
n \\
n-k+1
\end{array}\right)
\end{aligned}
$$


of the weighted $f O R-k$ can be easily defined easily as follows.

Definition 3. Given $f$ and $g$ two additive generators of a $t$-norm and its dual $t$-conorm, respectively, the weighted $k$ order fuzzy $A N D$ is defined by:

$\prod_{\mathbf{W}, \mathbf{v}}^{k}(\mathbf{x})=g^{(-1)}\left(\sum_{A \in \mathcal{P}_{k-1}} v_{A} g\left(f^{(-1)}\left(\sum_{j \in N \backslash A} w_{A, j} f\left(x_{j}\right)\right)\right)\right)$

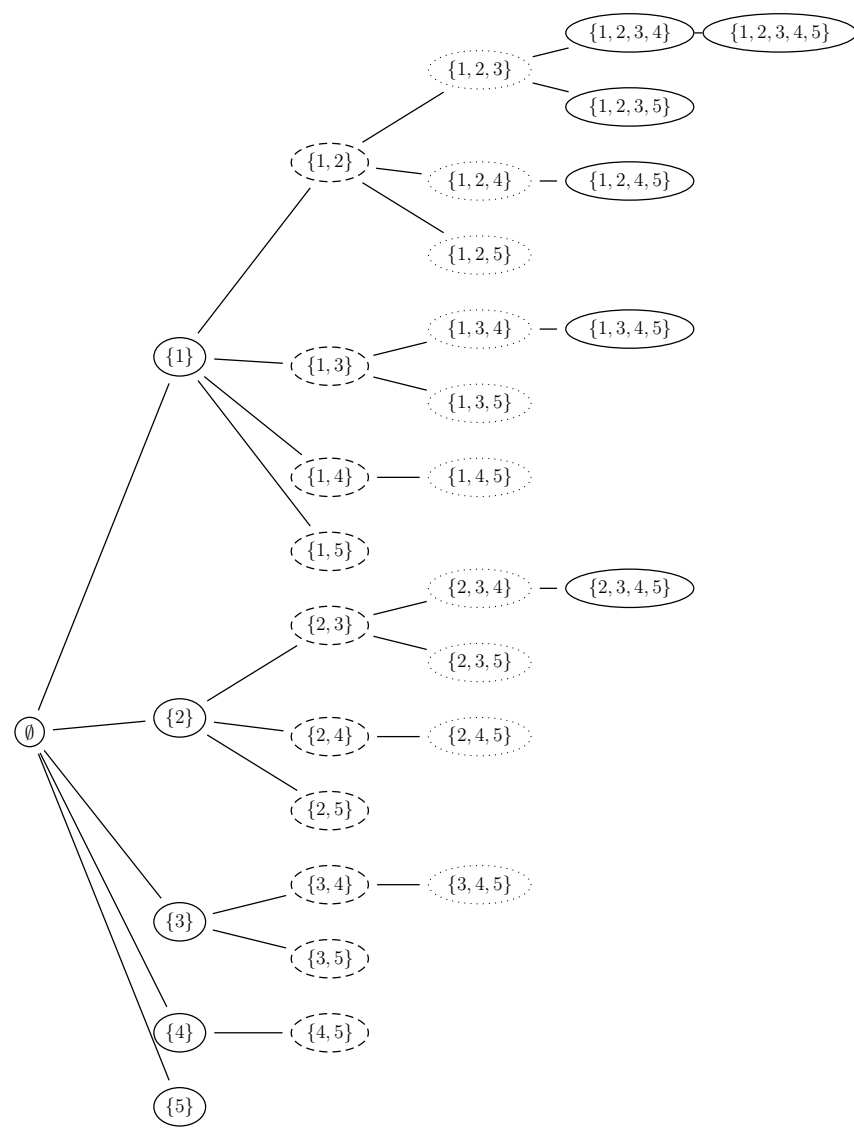

As requested, setting uniformly distributed weights in (11) and (12), i.e. constant weights $v_{A}$ and $w_{A, j}$ such as $\mathbf{v}$ and each row $\mathbf{w}_{\mathbf{A}}$ of $\mathbf{W}$ are normal, it is easy to show that the $k$-order fuzzy connectives fOR- $k$ (2) and $f A N D-k$ (4) are retrieved. More generally, even when not setting uniform weights, the properties of monotony and symmetry, as well as boundary conditions, are satisfied.

As mentioned in Section III, the $k$-order fuzzy connectives have been used in practical problems of pattern recognition like reject option, cluster validity or feature selection. The use of the weighted operators may improve the overall performances by specifying weights according to some criteria on the data to be analyzed.

\section{B. Examples}

We provide an illustrated example of weighting $k$-order fuzzy connectives with $n=5$ and $k=3$. A lattice representation of the different parts of $\mathcal{P}$ is given in Fig. 2, where dashed ellipsoids correspond the different $A \in \mathcal{P}_{k-1}$ and dotted ellipsoids to the subsets of $N$ where $j \in N \backslash A$ is acting. Weights have to be specified for subsets of cardinality $k-1=2$ (by $\mathbf{v}$ ) and for each of them, for subsets of cardinality $n-k+1=3$ (by $\mathbf{w}_{\mathbf{A}}$ ).

In Table III, we give some corresponding examples of randomly generated $\mathbf{v}$ and $\mathbf{W}$. One can see that the normal constraints, required to use additive generators, are satisfied for $\mathbf{v}(0.13+0.14+\ldots+0.15=1)$ and each $\mathbf{w}_{\mathbf{A}}$ (if $A=\{1,2\}$, then $w_{A, 3}+w_{A, 4}+w_{A, 5}=0.35+0.24+0.41=1$ ). The way the weights can be set with respect to an application is out of the scope of this paper and we invite the interested reader to refer to [14] to see some propositions, e.g. the minimization of an objective function to fit incoming data.

In Fig. 3, we give the isolevels of weighted $k=2$ order fuzzy connectives based on the standard norm couple $(\top, \perp)_{M}$ and the product one $(\top, \perp)_{P}$, where weights have been randomly generated. While the fundamental properties of the $k$-order fuzzy connectives are preserved, one can see that the shapes of each operator have been radically modified compared to the unweighted ones of Fig. 1, so that they can be adapted to various situations.

Fig. 2. A lattice representation of the parts of $\mathcal{P}$ under interest for $N=$ $\{1,2,3,4,5\}$

\section{Operations On FuZzy Sets}

Let $X$ be a nonempty universe and $A$ a fuzzy set of $X$ defined by its membership function $f_{A}: X \rightarrow I, x \mapsto f_{A}(x)$, where $I$ is a totally ordered set so that $(I, \leq)$ is a complete and totally ordered lattice. We restrict here to the case where $X$ is a denumerable set of $n$ elements, implying that a fuzzy set $A$ can be represented by the $n$-tuples $\left(\mathbf{x}, \mathbf{f}_{A}(\mathbf{x})\right)$ where $\mathbf{f}_{A}(\mathbf{x})=\left\{f_{A}\left(x_{1}\right), \ldots, f_{A}\left(x_{n}\right)\right\}$. The union and intersection of two fuzzy sets $A$ and $B$ are generally defined using a $t$-conorm and its dual $t$-norm, respectively. Then, for each $x \in X, A \cup B$ and $A \cap B$ are fuzzy sets whose membership functions are respectively $f_{A \cup B}(x)=f_{A}(x) \perp f_{B}(x)$ and $f_{A \cap B}(x)=f_{A}(x) \top f_{B}(x)$.

In order to interpret the presented $k$-order fuzzy connectives from a set-theoretic point of view, let us begin to restrict to three fuzzy sets $A, B$ and $C$ defined on $X$, and 
TABLE III

EXAMPLE OF WEIGHTS v AND W.

\begin{tabular}{|c|c|c|c|}
\hline Subset $A$ & $\mathbf{v}$ & $\mathbf{w}_{A}$ & $\begin{array}{c}\text { Relative indexes } \\
\quad j \in N \backslash A\end{array}$ \\
\hline \multirow{3}{*}{1,2} & \multirow{3}{*}{0.13} & 0.35 & $\{3\}$ \\
\hline & & 0.24 & $\{4\}$ \\
\hline & & 0.41 & $\{5\}$ \\
\hline \multirow{3}{*}{1,3} & \multirow{3}{*}{0.14} & 0.07 & $\{2\}$ \\
\hline & & 0.46 & $\{4\}$ \\
\hline & & 0.47 & $\{5\}$ \\
\hline \multirow{3}{*}{1,4} & \multirow{3}{*}{0.03} & 0.34 & $\{2\}$ \\
\hline & & 0.52 & $\{3\}$ \\
\hline & & 0.14 & $\{5\}$ \\
\hline \multirow{3}{*}{1,5} & \multirow{3}{*}{0.14} & 0.45 & $\{2\}$ \\
\hline & & 0.46 & $\{3\}$ \\
\hline & & 0.09 & $\{4\}$ \\
\hline \multirow{3}{*}{2,3} & \multirow{3}{*}{0.10} & 0.13 & $\{1\}$ \\
\hline & & 0.27 & $\{4\}$ \\
\hline & & 0.60 & $\{5\}$ \\
\hline \multirow{3}{*}{2,4} & \multirow{3}{*}{0.01} & 0.28 & $\{1\}$ \\
\hline & & 0.60 & $\{3\}$ \\
\hline & & 0.12 & $\{5\}$ \\
\hline \multirow{3}{*}{2,5} & \multirow{3}{*}{0.04} & 0.67 & $\{1\}$ \\
\hline & & 0.15 & $\{3\}$ \\
\hline & & 0.18 & $\{4\}$ \\
\hline \multirow{3}{*}{3,4} & \multirow{3}{*}{0.08} & 0.39 & $\{1\}$ \\
\hline & & 0.05 & $\{2\}$ \\
\hline & & 0.56 & $\{5\}$ \\
\hline \multirow{3}{*}{3,5} & \multirow{3}{*}{0.18} & 0.42 & $\{1\}$ \\
\hline & & 0.21 & $\{2\}$ \\
\hline & & 0.37 & $\{4\}$ \\
\hline \multirow{3}{*}{4,5} & \multirow{3}{*}{0.15} & 0.19 & $\{1\}$ \\
\hline & & 0.56 & $\{2\}$ \\
\hline & & 0.25 & $\{3\}$ \\
\hline
\end{tabular}

to focus on the most interesting case $^{6} k=2$. The 2 -order ${ }^{6}$ for $k=1$ or 3 , we have $\stackrel{1}{\top}=\top, \stackrel{3}{\top}=\perp, \stackrel{1}{\perp}=\perp$ and $\stackrel{3}{\perp}=\top$
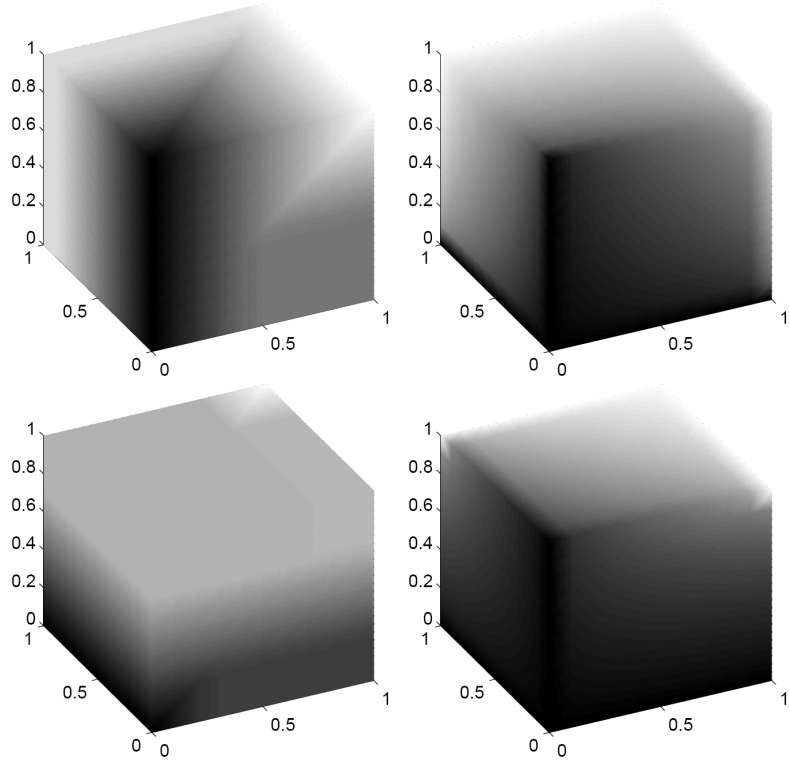

Fig. 3. Weighted fOR-2 (top) and fAND-2 (bottom) operators based on standard (left) and product (right) norm couples.

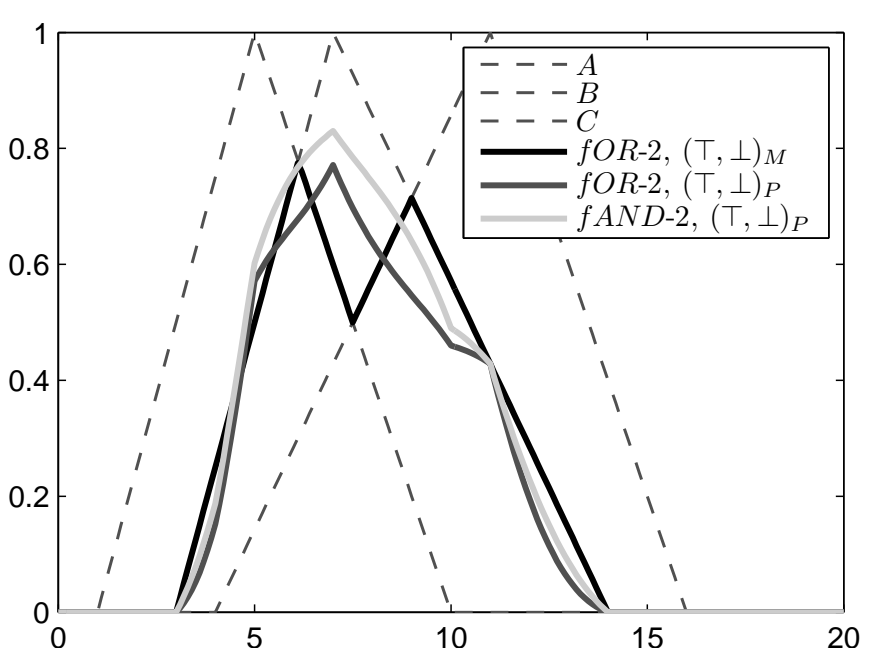

Fig. 4. Membership functions of the 2-order union and intersection of three triangular-shaped fuzzy sets $A, B$ and $C$.

fuzzy union is defined by $(A \cup B) \cap(A \cup C) \cap(B \cup C)$ and can be obtained thanks to the $f O R-2$ operator, which is just the conjunctive combination of subsets obtained by the disjunctive combination of all possible sets except $k-1$ ones. Alternatively, the 2-order fuzzy intersection is defined by $(A \cap B) \cup(A \cap C) \cup(B \cap C)$ and can be obtained thanks to the $f A N D-2$ operator.

Examples of these 2-order operation on three fuzzy sets whose membership functions are triangular ones are given in Fig. 4 for the standard norm couple $(T, \perp)_{M}$ and the product norm one $(\top, \perp)_{P}$. It is worth noting that the 2-order fuzzy union and the 2-order fuzzy intersection are the same set for $(\top, \perp)_{M}$ as one could expect from properties of the operators: when three values are to be aggregated, the $2^{\text {nd }}$ lowest value is also the $2^{\text {nd }}$ highest one. Note also that, although the 2-order fuzzy intersection and the 2-order fuzzy union are not equal, their membership function present a relatively similar shape: increasing until the second fuzzy set reaches its kernel, and decreasing afterward. From this figure, one can finally see that the membership functions of both 2-order fuzzy connectives are null on regions where there is only one support.

For more three fuzzy sets, the principle is the same: the $k$-order union is defined by the intersections of the unions of all the combination of fuzzy sets, excluding in each term $k-1$ fuzzy sets, and the previous remarks still remain. For instance, computing $k$-order fuzzy connectives on regions where $k-1$ supports of fuzzy sets are defined will results in an empty subset.

Our final proposition addresses the problem of quantifying if a fuzzy set $A$ has exactly $k$ elements using the proposed connectives. Let us recall the definition by Ralescu, where the author implicitly consider that having $k$ elements is 
equivalent to having $k$ high elements:

Definition 4 ([15]). The cardinality of a fuzzy set $A$, denoted $\operatorname{card}(A)$, is a fuzzy subset $\{0,1, \ldots, n\}$ with $\operatorname{card}(A(k))$ being the possibility to which $A$ has exactly $k$ elements, $0 \leq k \leq n$.

Proposition 2. Given a triangular norm couple $(T, \perp)$, the fuzzy cardinality of a fuzzy set $A$ is given by

$$
\overline{\operatorname{card}}(A(k))=\left(\stackrel{k}{\perp} \mathbf{f}_{A}(\mathbf{x})\right) \top\left(1-\stackrel{k+1}{\perp} \mathbf{f}_{A}(\mathbf{x})\right)
$$

The notation $\overline{\operatorname{card}}(A(k))$ is introduced to distinguish the operator which quantifies if $k$ elements are high, from $\operatorname{card}(A(k))$, the one which evaluate if there are $k$ low elements, and that we will call anticardinality. Note that if the standard norms $(\top, \perp)_{M}$ are taken, then (13) matches the proposition of Ralescu in [15], i.e. $f_{A}\left(x_{(k)}\right) \wedge\left(1-f_{A}\left(x_{(k+1)}\right)\right)$ if we assume that $(\cdot)$ is a permutation on $\mathbf{x}$ such as $f_{A}\left(x_{(1)}\right) \geq f_{A}\left(x_{(2)}\right) \geq \cdots \geq f_{A}\left(x_{(n)}\right)$.

Definition 5. The anti-cardinality of a fuzzy set $A$, denoted $\operatorname{card}(A)$ is a fuzzy subset $\{0,1, \cdots, n\}$ with $\underline{\operatorname{card}}(A(k))$ being the possibility to which $A$ has exactly low $k$ elements, $0 \leq k \leq n$.

Proposition 3. Given a triangular norm couple $(\top, \perp)$, the fuzzy anti-cardinality of a fuzzy set $A$ is given by

$$
\begin{aligned}
\underline{\operatorname{card}}(A(k)) & =\left(1-\stackrel{k}{\top} \mathbf{f}_{A}(\mathbf{x})\right) \top\left(\stackrel{k+1}{\top} \mathbf{f}_{A}(\mathbf{x})\right) \\
& =1-\left(\left({ }^{\frac{k}{\top}} \mathbf{f}_{A}(\mathbf{x})\right) \perp\left(1-\stackrel{k+1}{\top} \mathbf{f}_{A}(\mathbf{x})\right)\right)
\end{aligned}
$$

If $A$ is a crisp set with $c$ elements, the ordered $\mathbf{f}_{A}(\mathbf{x})$ is $\{1, \ldots, 1,0, \ldots, 0\}$ composed of $(n-c)$ zeros and $c$ ones. For all triangular norms, we have:

$$
\begin{gathered}
\overline{\operatorname{card}}(A(k))= \begin{cases}1 & \text { if } k=c \\
0 & \text { otherwise }\end{cases} \\
\underline{\operatorname{card}}(A(k))= \begin{cases}1 & \text { if } k=n-c \\
0 & \text { otherwise }\end{cases}
\end{gathered}
$$

which satisfies the given definitions.

A clear connection to specificity measures, or more generally to amount of information measures (e.g. entropy in probability theory, granularity in fuzzy theory, and measure of uncertainty in possibility theory) is obtained by setting $k$ to 1. However, the proposed operators are more general, since they can handle much more desiderata.

\section{CONCLUSION}

In this paper, the $k$-order fuzzy connectives are presented. We propose to use these operators in order to evaluate whether exactly $k$ values are high or low, respectively.
Some examples are given to illustrate the behavior of the operators, and how to compute them. Weighted $k$-order fuzzy connectives are also introduced for the purpose of modelizing dependency between the values to be aggregated if any. The use of the proposed $k$-order connectives to define new settheoretic operations on fuzzy sets is also proposed. Finally, the definition of a generalized fuzzy cardinality, representing to what extent a fuzzy set contains exactly $k$ high or low values, is proposed.

Among the perspectives we have in mind, let us mention the linear or exponential combination of $k$-order fuzzy connectives, as well as the uninorm like combination. We also plan to apply our propositions on real world problems such as pattern recognition and image analysis. Finally, a complete characterization of the involved weights in terms of their Moebius transform and Shapley value is under study.

\section{REFERENCES}

[1] M. Grabisch, J. Marichal, R. Mesiar, and E. Pap, Aggregation Functions, ser. Encyclopedia of Mathematics and its Applications. Cambridge University Press, 2009, no. 127.

[2] A. Ralescu, D. Ralescu, and K. Hirota, "Evaluation of fuzzy quantified expressions," in Fuzzy Logic in Artificial Intelligence, LNCS 1566, 1997, pp. 234-244.

[3] R. Yager, "Noble reinforcement in disjunctive aggregation operators," IEEE Transactions on Fuzzy Systems, vol. 11, no. 6, pp. 754-767, 2003.

[4] - "Measures of specificity over continuous spaces under similarity relations," Fuzzy Sets and Systems, vol. 159, no. 17, pp. 2193-2210, 2008.

[5] K. Menger, "Statistical metrics," Proc. National Academy of Science USA, vol. 28, no. 12, pp. 535-537, 1942.

[6] E. Klement, R. Mesiar, and E. Pap, Triangular Norms, T. in Logic, Ed. Kluwer Academic, 2000.

[7] L. Mascarilla, M. Berthier, and C. Frélicot, "A k-order fuzzy or operator for pattern classification with k-order ambiguity rejection," Fuzzy Sets and Systems, vol. 159, no. 15, pp. 2011-2029, 2008.

[8] H. Le Capitaine and C. Frélicot, "A cluster validity index combining an overlap measure and a separation measure based on fuzzy aggregation operators," submitted to IEEE Transactions on Fuzzy Systems, 2010.

[9] L. Mascarilla and C. Frélicot, "A class of reject-first possibilistic classifiers based on dual triples," in Proceedings of the 9th International Fuzzy Systems Association World Congress, 2001, pp. 743-747.

[10] G. Beliakov and T. Calvo, "Construction of aggregation operators with noble reinforcement," IEEE Transactions on Fuzzy Systems, vol. 15, no. 6, pp. 1209-1218, 2007.

[11] D. Dubois and H. Prade, "A review of fuzzy set aggregation connectives," Information Sciences, vol. 36, pp. 85-121, 1985.

[12] T. Calvo and R. Mesiar, "Weigthed triangular norms-based aggregation operators," Fuzzy Sets and Systems, vol. 137, no. 1, pp. 3-10, 2003.

[13] M. Grabisch, "k-order additive discrete fuzzy measures and their representation," Fuzzy Sets and Systems, vol. 92, no. 2, pp. 167-189, 1997.

[14] G. Beliakov, "Construction of aggregation functions from data using linear programming," Fuzzy Sets and Systems, vol. 160, no. 1, pp. 65-75, 2009.

[15] D. Ralescu, "Cardinality, quantifiers, and the aggregation of fuzzy criteria," Fuzzy Sets and Systems, vol. 69, pp. 355-365, 1995. 